\title{
Inactivated Poliovirus Vaccine: The Fog of Uncertainty is Lifting!
}

\author{
T ЈАСОВ ЈоHN \\ Thekkekara, 439 Civil Supplies Godown Lane, Kamalakshipuram, Vellore, TN 632002. \\ tjacobjohn@yahoo.co.in
}

$\mathrm{I}$ ndia achieved zero transmission of wild polioviruses (WPVs) in 2011. On 25 February, 2012, the World Health Organization (WHO) removed India from the list of WPV-endemic countries. Formal certification of eradication requires three years without WPVs, expected in the first quarter of 2014. Then we have to advance to the second phase of polio eradication, for eliminating vaccine polioviruses (VPVs) [1].

Albert Sabin derived VPVs by attenuating WPVs. Two major phenotypic changes were brought about by genetic variations: loss of neurovirulence and reduction in 'force of infection'. Force of infection determines the efficiency of transmission between hosts: VPVs are less efficient in transmission than WPVs. Polioviruses, with single stranded positive sense RNA genome, acquire point mutations during replication. As a result, VPVs tend to revert genetically during cycles of virus replication. In serial replication in humans, VPVs de-attenuate and become 'vaccine-derived polioviruses' (VDPVs) [2]. If all children are fully vaccinated with OPV, VDPVs will not emerge but the price to pay will be the occasional vaccineassociated paralytic polio (VAPP). So, OPV has to be withdrawn, when the stray VDPVs will circulate unchecked among children, become 'circulating VDPVs' (cVDPVs) and replace the eradicated WPVs. The emergence of cVDPVs should therefore be preempted at all costs [1]. We already have the necessary tool of intervention, the inactivated poliovirus vaccine (IPV) developed by Jonas Salk.

Now the contours of eradication phase 2 are clear: introduce IPV, achieve high coverage, discontinue OPV and maintain clinical and virological surveillance [1]. These steps will have to be orchestrated globally in order to conclude polio eradication. WHO is planning to discontinue VPVs in stages, starting with withdrawal type 2 by switching from trivalent to bivalent OPV (bOPV, containing types 1 and 3 ), since WPV type 2 was eradicated in 1999. In preparation for this shift, IPV needs to be introduced universally. Where schedule-based vaccination will not achieve high coverage, to begin with IPV may have to be given in campaigns. After WPV 1 and 3 are eradicated, bOPV will also be withdrawn.
Many countries currently use IPV-containing pentavalent vaccine with DPT with acellular pertussis (aP) component and Haemophilus influenza b (Hib) conjugate vaccine or hexavalent vaccine containing hepatitis $B$ vaccine (HB) also. Hexavalent is ideal for India since we have $\mathrm{HB}$ and $\mathrm{Hib}$ in our Universal Immunisation Programme(UIP). If hexavalent with wP can be made, that will be even better.

Investigators from Delhi and Vellore have conducted clinical trials of a pentavalent vaccine manufactured in France $[3,4]$. In primary series in UIP schedule $(6,10,14$ weeks of age), immune responses were excellent to all components, quite similar to those obtained in France using a 2, 3, 4 months schedule [3]. In this issue, they report on a booster dose given in the second year of life; the seroresponses were excellent - achieving very high antibody levels to each antigen [4]. Taken together, they show that IPV-containing combination vaccines can immunize well when given according to our UIP schedule for DPT - primary series at 6, 10, 14 weeks and a booster in the second year of life. We now await the revision of national policy for the introduction of IPV in UIP.

Competing interests: None stated; Funding: Nil.

\section{REFERENCES}

1. John TJ, Vashishtha VM. Eradication of vaccine polioviruses: why when and how? Indian J Med Res. 2009;130:491-4.

2. Kew O, Morris-Glasgow V, Landaverde M, Burns C, Shah $\mathrm{J}$, Garib Z, et al. Outbreak of poliomyelitis in Hispaniola associated with circulating type 1 vaccine-derived poliovirus. Science. 2002;296:356-9.

3. Dutta AK, Varghese VP, Pemde HK, Mathew LG, Ortiz E. Immunogenicity and safety of a pentavalent diphtheria, tetanus, acellular pertussis, inactivated poliovirus, Haemophilus influenzae type $\mathrm{b}$ conjugate combination vaccine (Pentaxim) with hepatitis B vaccine. Indian Pediatr. 2009;46:975-82.

4. Dutta AK, Varghese VP, Pemde HK, Mathew LG, Ortiz E. Immunogenicity and safety of a DTaP-IPV/PRP $\sim$ vaccine booster dose during the second year of life in Indian children primed with the same vaccine. Indian Pediatr. 2012; 49:793-8. 